\title{
Portable x-ray fluorescence spectrometer for coating thickness measurement
}

\author{
Alain Carapelle, ${ }^{\text {a) }}$ Karl Fleury-Frenette, and Jean-Paul Collette \\ Centre Spatial de Liège (CSL), University of Liège, Avenue de Pre-Ally, Liege Science Park, 4031 Angleur, \\ Belgium \\ Henri-Pierre Garnir \\ Institut de Physique Nucléaire, Atomique et de Spectroscopie (IPNAS), University of Liege, 15 Allée du Six \\ Août, Sart Tilman, 4031 Angleur, Belgium \\ Philippe Harlet \\ Arcelor-Mittal Research Liège, Boulevard de Colonster, B57, 4000 Liège, Belgium
}

(Received 4 July 2007; accepted 16 November 2007; published online 18 December 2007)

\begin{abstract}
A handheld x-ray spectrometer has been realized and tested. The purpose of the device is to measure the thickness of coated samples in the range of $1-1500 \mathrm{~nm}$ in an industrial environment. Accuracy of $\sim 3 \%$ has been achieved in this range with a measurement time of $1 \mathrm{~min}$. Automated software has been implemented to allow utilization by a nonspecialist operator. An automated calibration procedure, based on measurements of reference samples, is used. (C) 2007 American Institute of Physics. [DOI: 10.1063/1.2822613]
\end{abstract}

\section{INTRODUCTION}

In the frame of its optical coating facilities, the Centre Spatial de Liège (CSL) needed a portable device for the measurement, in the field, of coating thickness. The device had to be handheld to allow its utilization near different coating facilities. Another requirement was its ease of use; it had to be used by a nonspecialist operator. Therefore the operation of the device had to be as easy as possible.

The utilization of $\mathrm{x}$-ray fluorescence (XRF) has been largely adapted to several applications (for example, tabletop microbeam imaging, ${ }^{1}$ miniature setup ${ }^{2}$ ).

Thickness measurement by XRF is also routinely used in tabletop laboratory devices (for example, Fisherscope X-Ray form Fisher Technology, X-Strata960 from Oxford Instruments). An XRF spectrometer has also been developed for particular applications such as archeometry ${ }^{3,4}$ or metal alloy identification (for example, Thermo-NITON XLt series). But there is no available handheld device using XRF measuring the thickness of coatings in the range of $1-1500 \mathrm{~nm}$.

\section{INSTRUMENT DESCRIPTION}

The principle used by the device is the following: an $\mathrm{x}$-ray tube irradiates the sample and $\mathrm{x}$-ray fluorescence occurs; a detector counts incoming $\mathrm{x}$-ray photons and measures their energies. The $\mathrm{x}$-ray fluorescence spectrum is recorded and analyzed. Figure 1 shows a functional schematic of the device.

The design of the instrument is classical for an XRF instrument; the novelties are its adaptation for automatic thickness measurement and its portability. Figure 2 shows the device with its onboard computer.

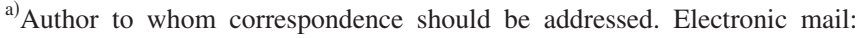
a.carapelle@ulg.ac.be.
}

The weight of the device, including its computer, is less than $4 \mathrm{~kg}$. The $\mathrm{x}$-ray tube has a maximum voltage of $35 \mathrm{kV}$, a maximum current of $0.1 \mathrm{~mA}$, and a spot size of $\sim 0.4$ $\times 0.9 \mathrm{~mm}$ (Manufacturer Moxtek, model TUB00020-1 with $\mathrm{Ag}$ anode). The detector is a Peltier cooled silicon drift detector $^{5}$ with a full width at half maximum of $135 \mathrm{eV}$ (measured on $\mathrm{Mn} K \alpha$ line produced by a ${ }^{55} \mathrm{Fe}$ radioactive source). The preamplifier and amplifier have been developed specifically for this device. The software has been written for the utilization by a nonspecialist operator (simple push button interface).

In our case the coating layer and substrate compositions were known. Besides, reference samples were available. Thanks to these particular conditions, there was no need to identify the atoms and, therefore, the device's software was very simple because no identification algorithm was required.

As we knew the coating composition, we knew its fluorescence's energies. Therefore we chose a particular fluorescence line regarding three criteria.

- The fluorescence yield of this particular line had to be high (this is partially dependent on the spectrum of the x-ray excitation tube and hence on its high voltage).

- The fluorescence line energy position had to be near the maximum of the energy response curve of the detector.

- The fluorescence line energy position had to be not too close to a fluorescence line of the substrate of the coating.

\section{SOFTWARE DESCRIPTION}

When the appropriate line was chosen, we calculated its yield in the spectrum by summing the peak area in the spectrum and removing the background. Figure 3 illustrates the principle of this calculation. 


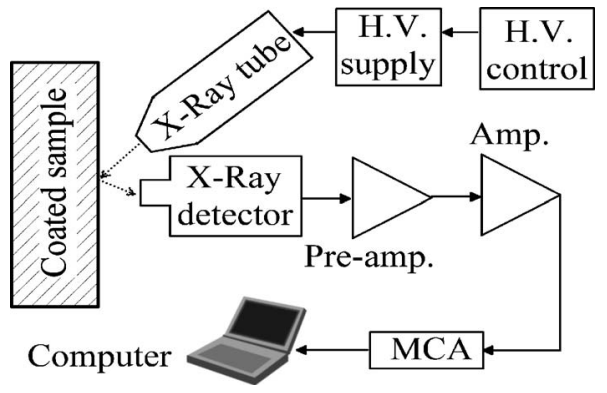

FIG. 1. Instrument schematic description.

If the energy of the chosen line was $E_{i}$, we chose a region of interest (ROI) based on the detector resolution and the natural linewidth, between $E_{1}$ and $E_{2}$. We measured the intensity of the signal of this particular line by removing the background modelized by an area of a trapezoidal shape. Therefore the intensity $I$ was given by

$$
I=\sum_{E=E_{1}}^{E_{2}} I(E)-\left(\frac{I_{1}+I_{2}}{2}\right)\left(E_{2}-E_{1}\right),
$$

where $I(E)$ is the intensity, for this particular channel [as no energy calibration was needed, the $x$-axis unit was the channel number of the multichannel analyzer (MCA)]; therefore the $\sum_{E=E_{1}}^{E_{2}} I(E)$ was the sum of the counts in each channel between energies $E_{1}$ and $E_{2} . I_{1}$ and $I_{2}$ are, respectively, the intensities of the channel of energy $E_{1}$ and $E_{2}$.

Ideally, the intensity $I$ would have been proportional to the thickness; in reality, a kind of "saturation" phenomenon occurs, mainly due to two causes: self-absorption of the measured layer ${ }^{6}$ and pileup and sum peaks. ${ }^{7}$ These two phenomena lead to a nonlinear response curve that can be fitted with good approximation with a second order polynomial curve,

$$
T=a I^{2}+b I+c,
$$

where $T$ is the thickness of the deposited layer and $I$ the intensity of the considered fluorescence peak calculated with Eq. (1). The coefficients $a, b$, and $c$ are calculated by a classical fitting procedure.

For the calibration of our particular setup we took a series of spectra of samples with a deposited layer of known thickness and calculate the measured intensity of the chosen peak using Eq. (1) and we fitted the intensity as function of the thickness with a second order polynomial curve. The curve obtained was used as a calibration curve.

Some thickness measurements of very thin layers (1 or $2 \mathrm{~nm}$ ) were more difficult to carry out due to spectroscopy artifacts (detector resolution degradation due to increase of

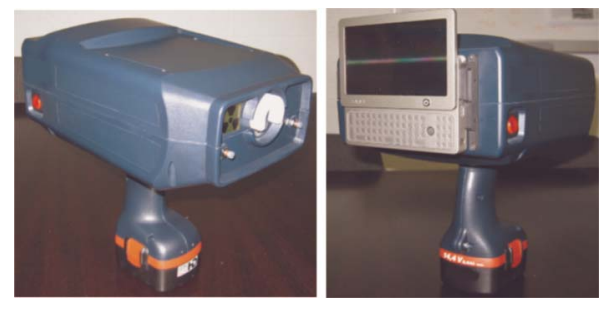

FIG. 2. (Color online) Pictures of the instrument.

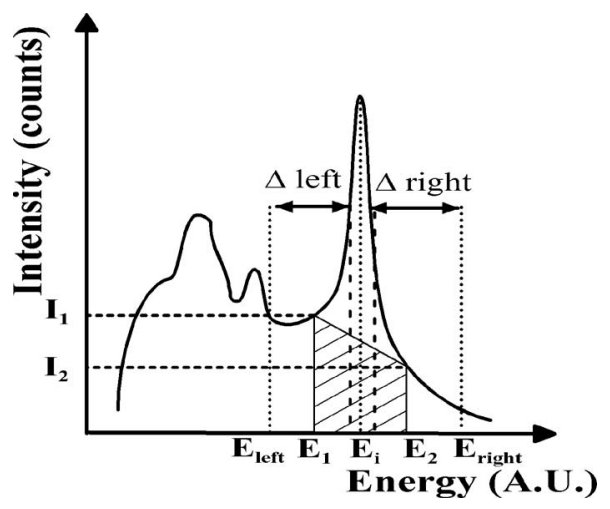

FIG. 3. Intensity calculation and ROI choice.

temperature for long measurement series, ${ }^{8}$ resolution degradation due to high count rate, ${ }^{9}$ MCA nonlinearity, small S/N ratio) and due to experimental conditions (sample position reproducibility, layer deposition inhomogeneity). In those cases, the ROI surrounding the considered peak had to be carefully set in the software. An automated subroutine of the software has been implemented to choose the best ROI in a given "extended" range from $E_{\text {left }}$ to $E_{\text {right }}$ (see Fig. 3 ). This subroutine tried all the possible values for $E_{1}$ and $E_{2}$ and found the best ones, called, respectively, BestE $E_{1}$ and $B_{e s t} E_{2}$. Details of the algorithm of this subroutine can be found as an extended caption of Fig. 3 on EPAPS. ${ }^{10}$ At the end of the subroutine, the best ROI (with boundaries BestE $E_{1}$ and BestE $_{2}$ ) was used to calculate the intensity with Eq. (1). Thanks to the power of current computers, this subroutine only took a few seconds (less than $10 \mathrm{~s}$ on a handheld OQO 01 Windows XP computer with $1 \mathrm{GHz}$ processor) to compute optimum values for $E_{1}$ and $E_{2}$. This subroutine also worked well in the case of thick layer measurement; therefore it had been implemented in the device for all the measurements.

Practically, when a new kind of sample (new coating and new substrate) had to be measured, a first calibration phase was needed. For this phase a set of calibrated samples of known thickness was used, these calibrated samples were submitted to the device and the software first calculated the intensity of fluorescence for each sample using Eq. (1) and then proceeded to the parabolic fitting for Eq. (2). Then the software proceeded to ROI optimization. At the end of the process, a calibration curve was obtained and saved in the device. At that point, a measurement of sample of unknown thickness was possible by measuring the intensity and using the calibration curve to calculate the thickness. The calibration and measurement process had been made as simple as possible by the software. The only interaction with the operator is the input of the known thickness of calibrated samples during calibration phase.

After the calibration curve was saved, the operator had the possibility to recalibrate (in the case of important temperature change in the room or sample geometrical modification, for example).

\section{RESULTS}

We have made several dozens of measurements and calibration curve on various coatings and substrates. We have 


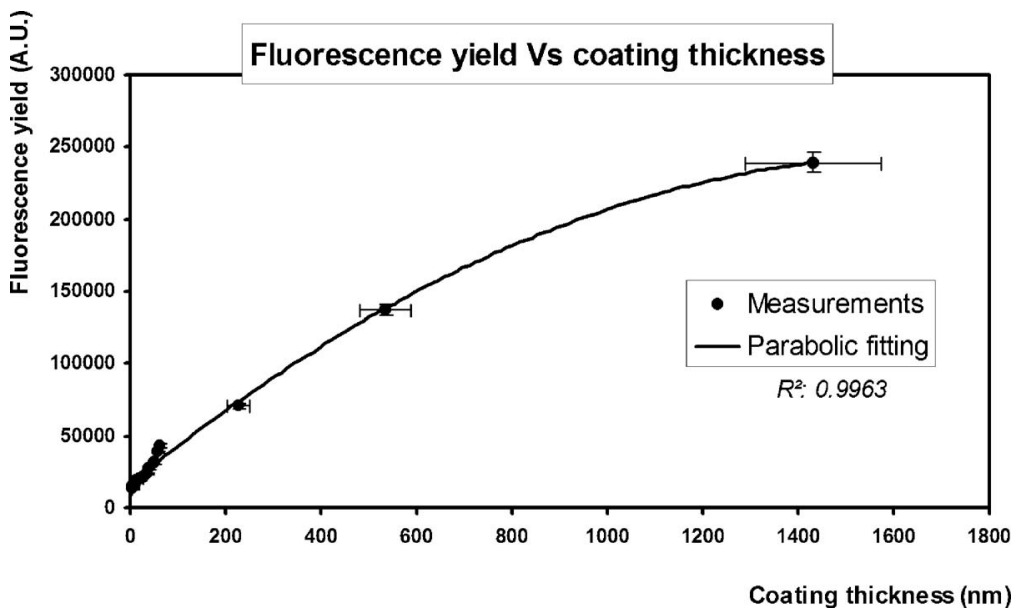

FIG. 4. Calibration curve for copper on silicon.

detailed copper layer thickness measurement on silicon wafer substrate. Spectroscopic ellipsometry and phase-shift interferometry were used in combination to measure the thickness. The intensity, calculated with Eq. (1), of the $K \alpha$ fluorescence line of the copper at $8.048 \mathrm{keV}$ was plotted versus the measured thickness to allow the fitting of the calibration curve. The results are given in Fig. 4 , where $R^{2}$ is the coefficient of determination.

The results illustrated are in good agreement with absorption-length value given in literature ${ }^{11}$ for the $K$-absorption edge of copper.

Thanks to its abilities to be used by a nonspecialist, the device is now used not only at the CSL coating facility but has been put into routine operation in a large industrial steel plant. It has been demonstrated that the device can measure accurately the following coatings: $\mathrm{Cu}, \mathrm{Al}, \mathrm{Mg}, \mathrm{Ti}, \mathrm{Cr}, \mathrm{S}, \mathrm{Ru}$ in the range of $1-2000 \mathrm{~nm}$. As explained in paragraph IV, the device is not calibrated in energy. Therefore, the positions of the used fluorescence line are given in MCA channel numbers and hence are only usable in our particular device.

For low $Z$ elements ( $\mathrm{Mg}, \mathrm{Al}$, and $\mathrm{S}$ ) measurements have been done under helium flushing to avoid fluorescence absorption by atmospheric Ar. When He flushing was used, the pressure and flux of He must be very stable to avoid measurement fluctuations.

Due to the $x$-ray tube intensity instabilities of $\pm 3 \%$ (data provided by Moxtek's characterization data sheet provided with each individual $\mathrm{x}$-ray tube, measurement based on the ratio of $\mathrm{Pb} L_{\beta} / \mathrm{Fe} K_{\alpha}$ fluorescence line intensity); the maximum measurements accuracy was, at best, $\sim \pm 3 \%$ (standard deviation). In our tests with a series of ten measurements on Ti layer of $1200 \mathrm{~nm}$ on an iron alloy sample the experimental statistical deviation obtained was $3.32 \%$.

\section{CONCLUSION}

Despites its simple principle, this device fulfills industrial needs in portable thin layer thickness measurement. Of course the system is not universal, the atomic composition of the substrate matrix and of the measured layer must be known and it must have x-ray fluorescence characteristics that meet the three requirements explained in paragraph II. Besides reference samples of known thickness must be available for calibration.

Further improvements are planned: continuous monitoring of incident $\mathrm{x}$-ray flux to override $\mathrm{x}$-ray tube natural fluctuation, implementation of advanced algorithm for $\mathrm{x}$-ray spectrum evaluation, ${ }^{12}$ reduction of size and weight by highly integrated and miniaturized electronic, replacement of amplifier and MCA by a digital signal processor.

The actual version of the device is commercially available ("Finder 100" sold by Walopt Company).

\section{ACKNOWLEDGMENTS}

We would like to thanks Arcelor-Mitall Research Liege and development center for its help for adapting the device to their industry requirements.

${ }^{1}$ A. Attaelmanan, S. Larsson, A. Rindby, P. Voglis, and A. Kuczumow, Rev. Sci. Instrum. 65, 7 (1994).

${ }^{2}$ C. Ribbing, M. Anderson, K. Hjort, and H. Lundqvist, Rev. Sci. Instrum. 74, 3423 (2003).

${ }^{3}$ A. Longoni, Nucl. Instrum. Methods Phys. Res. A 409, 407 (1998).

${ }^{4}$ D. Strivay, G. Weber, A. Marchal, M. Clar, and H.-P. Garnir, In Situ Non-Destructive Analysis and Testing of Museum Objects, Bratislava (Slovaquie), April 2005.

${ }^{5}$ P. Lechener, S. Eckbauer, R. Hartmann, S. Krisch, D. Hauff, R. Richter, H. Soltau, L. Struder, C. Fiorini, E. Gatti, A. Longoni, and M. Sampietro, Nucl. Instrum. Methods Phys. Res. A 377, 346 (1996).

${ }^{6}$ R. E. Van Grieken and A. A. Markowicsz, Handbook of X-Ray Spectrometry, Practical Spectroscopy Series Vol. 29, 2nd ed. (Dekker, New York, 2002) p. 366.

${ }^{7}$ Handbook of X-Ray Spectrometry Second Edition, edited by R. E. Van Grieken and A. A. Markowicsz, Practical Spectroscopy Series (Dekker, New York, 2002), Vol. 29, p. 243

${ }^{8}$ P. Lechener, C. Fiorini, R. Hartmann, J. Kemmer, N. Krause, P. Leutenegger, A. Longoni, H. Soltau, D. Stotter, R. Stotter, L. Struder, and U. Weber, Nucl. Instrum. Methods Phys. Res. A 458, 281 (2001).

${ }^{9}$ R. E. Van Grieken and A. A. Markowicsz, Handbook of X-Ray Spectrometry, Practical Spectroscopy Series Vol. 29, 2nd ed. (Dekker, New York, 2002) p. 232.

${ }^{10}$ See EPAPS Document No. E-RSINAK-78-063712. This document can be reached through a direct link in the online article's HTML reference section or via the EPAPS homepage (http://ww.aip.org/pubservs/epaps.html).

${ }^{11}$ B. L. Henke, E. M. Gullikson, and J. C. Davis, At. Data Nucl. Data Tables 54, 181 (1993).

${ }^{12}$ B. Vekemans, X-Ray Spectrom. 23, 278 (1994). 